\title{
Review of: "Immediate Impact of Yogic Breathing on Pulsatile Cerebrospinal Fluid Dynamics"
}

\author{
Vegard Vinje
}

Potential competing interests: The author(s) declared that no potential competing interests exist.

In this paper the authors studied CSF movement at the foramen magnum affected by yogic breathing using real-time phase contrast MRI. Subjects were asked to perform several breathing protocols from spontaneous breathing to various types of deep breathing. They found that CSF velocities were altered in yogic versus spontaneous breathing with a $28 \%$ increase in CSF velocity during deep abdominal breathing. Under normal conditions, cardiac pulsations dominate CSF velocities. However during deep abdominal breathing the contribution from cardiac and respiratory components are similar.

The paper is well written, and I do not have any major concerns. However, I do have some minor comments below.

Comments:

1) Net flow has been reported in some studies e.g. (https://pubmed.ncbi.nlm.nih.gov/30443816/). Was net flow considered at all in this study? Was a postprocessing average routine performed? (E.g. shift the signal to zero net flow). Or calibration of a ROI in static tissue to confirm zero net flow there (similar to the confirmation of no pulsatility in static tissue).

2) One subject had a higher breathing frequency during slow breathing than during spontaneous breathing, at least $50 \%$ higher. This is a surprising result, and may be commented upon.

3) It is mentioned several (at least three) times that the report is the first in its type w.r.t yogic breathing. I think this is a bit misleading, although it is the first to mention yogic breathing whilst reporting effects of breathing on CSF dynamics. Previous papers (e.g. both Dreha-Kulaczewski papers) have reported effect of forced breathing/breathing protocols compared to normal/spontaneous breathing. The new aspect of this study, compared to previous reports, seem to be that the authors compare several different breathing protocols. From previous reports we knew that 2 different breathing patterns (e.g. "normal" vs "forced", (Dreha-Kulaczewski)) would inducedifferent CSF velocities. This report expands this knowledge with more details.

My suggestion would be to be more careful with these sentences about being the first study. I think the 
study is interesting enough without specifying this anyways.

4) In the discussion p37 first paragraph: May include a citation on increased pulastile CSF dynamics during sleep to Fultz et al.

I look forward to reading more about the long-term effects of these protocols. 